\title{
Physio- and chemo-dual crosslinking toward thermo- and photo-response of azobenzene-containing liquid crystalline polyester
}

\author{
Hai-Yi Zhong ${ }^{1,2}$, Li Chen $^{1 *}$, Xiao-Min Ding ${ }^{1}$, Rong Yang ${ }^{3}$ and Yu-Zhong Wang ${ }^{1 *}$
}

\begin{abstract}
Combining the stability of chemical crosslinking and the processability of physical crosslinking is a well-established strategy to design new materials with desirable stimuli-responsive properties. Herein, a series of azobenzenebased thermotropic liquid crystalline polyesters were synthesized by introducing mesogenic dial named 4,4'-bis(6-hydroxyhexyloxy)azobenzene (BHHAB), 2-phenylsuccinic acid (PSA), and different contents of 1,2,3-propanetricarboxylic acid (PTA) as the chemical crosslinker. All these polyesters showed good thermal stability and smectic liquid crystalline phase. Wide-angel X-ray diffraction (WAXD) and the fluorescence emission spectra confirmed the existence of $\pi-\pi$ stacking interactions as the physical crosslinking in the polymer chains, particularly at the lower content of PTA. However, when the PTA content increased, the chemical crosslinking changed the chain conformation, and thus the intensity of physical crosslinking slackened gradually. Combining the physical and chemical crosslinking, these polyesters showed the thermoplastic processability, thermal shape memory, heat-assisted healing and photoresponsive behaviors. Taking advantages of these features, these multiple stimuli-responsive polymers can bring more chances for smart materials such as soft actuator.
\end{abstract}

Keywords: liquid crystalline polymer, crosslinking, shape memory, photo-response, self healing

\section{INTRODUCTION}

Crosslinked liquid crystalline polymers (CLCPs) are developed on the basis of the liquid crystalline polymers (LCPs). Through the moderate density of crosslinking, CLCPs display elasticity either in isotropic state or in liquid crystal state [1,2]. Attractively, as typical polymer exhibiting the ability to change its shape reversibly after the application of a certain external stimulus [3], CLCPs combine the properties of polymeric elastomers (entropy elasticity) with liquid crystals (self-organization) [4]. Since de Gennes predicted the shape memory properties of CLCPs in 1975 [5], a large amount of CLCPs with different liquid crystalline phases and network structures have been synthesized and characterized, including mainchain smectic [6,7], side-chain smectic $[8,9]$ and nematic $[10,11]$ CLCPs, According to the different structures designed in the CLCPs, the materials can be responsive to different stimuli including heat, light, electricity and so on [12-17].

For shape memory polymers, the fixing phase, such as crystalline phase, intermolecular forces in polymer chains, crosslinking, are the important factors to determine the constant shape of materials [18-21]. As for CLCPs, the crosslinking can be regarded as the fixing phase which ensures the translation of microscopic changes into macroscopic motions and the repeatability of the actuation. In a normal condition, the chemical crosslinking in the CLCPs plays an extremely important role in their shape memory behavior because of its enough strength and stability. However, the chemical crosslinking generally occurs during the synthesis process and could not be changed anymore once synthesized $[22,23]$, which might sacrifice the processability and restrict the practical application of the CLCPs.

In recent years, scientists have paid much attention on finding new modes of crosslinking to replace the normal chemical crosslinking such as the exchangeable covalent

\footnotetext{
${ }^{1}$ Center for Degradable and Flame-Retardant Polymeric Materials, College of Chemistry, State Key Laboratory of Polymer Materials Engineering, National Engineering Laboratory of Eco-Friendly Polymeric Materials (Sichuan), Sichuan University, Chengdu 610064, China

${ }^{2}$ College of Pharmacy, Guangxi University of Chinese Medicine, Nanning 530299, China

${ }^{3}$ School of Materials Science and Engineering, Changzhou University, Changzhou 213000, China

" Corresponding authors (emails: 1.chen.scu@gmail.com (Chen L); yzwang@scu.edu.cn (Wang YZ))
} 

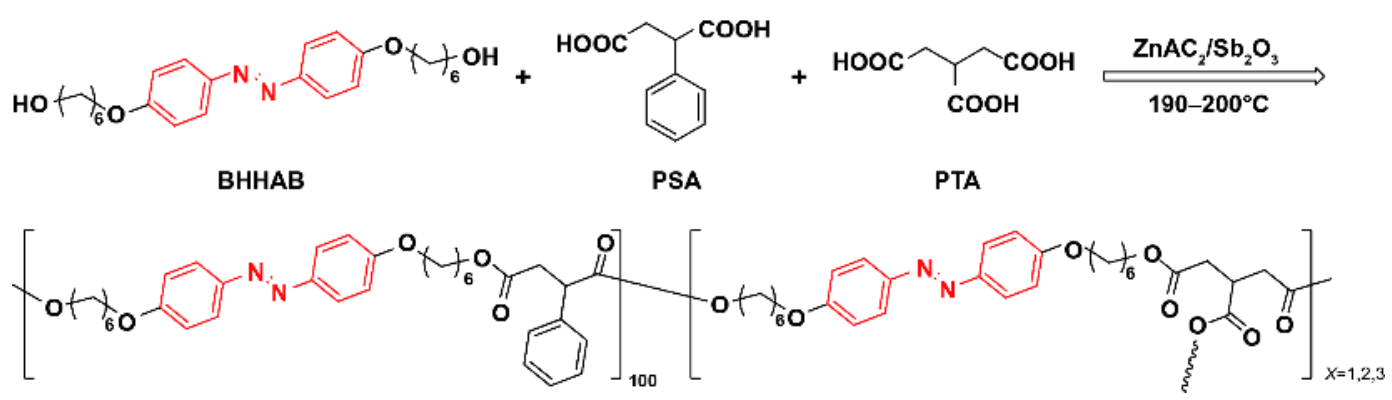

Scheme 1 Synthetic route and chemical structures of PBHPS- $x \%$ PTA, where 100 and $x$ denote the molar ratio with regard to PSA, not the block length.

bonds or the post-crosslinking method etc. [24-29], where different secondary bonds might be another answer, including hydrogen bond, $\pi-\pi$ interaction, coordination linkage, and so on. For example, Ikeda et al. [30] prepared azo based liquid crystalline films using hydrogen bond as crosslinking points, obtaining the reversible bending/unbending properties under UV/visible light as well. Zhang et al. [31] synthesized azobenzene main-chain LCPs via Michael addition polymerization and then studied the photomechanical effects of their supramolecular hydrogen-bonded fibers. Chen et al. [32] synthesized a side-chain LCP which showed good thermal shape memory property with the polymer chain columns acting like physical crosslinks. In our previous studies [33-38], two main-chain LCPs with inter-/intra-molecular $\pi-\pi$ interaction as crosslinking points were designed and synthesized, and the resulting good shape memory behaviors proved that the $\pi-\pi$ interaction could also be an effective modes to build stimuli responsive CLCPs. In summary, physical crosslinking can solve the processibility effectively caused by chemical crosslinking, but these weak intermolecular interactions may be influenced easily by the movement of the polymer chains in some conditions, which also restricted the application of materials.

Architecturally, combining the stability of chemical crosslinking and the processibility of physical crosslinking might be a good method to endow materials with better comprehensive properties $[39,40]$. Thus, in this study, a series of azobenzene-containing liquid crystalline polyesters with slight crosslinking were synthesized as shown in Scheme 1. Herein the phenyl group of 2-phenylsuccinic acid (PSA) provided the $\pi-\pi$ interaction in the polymer chain as the physical crosslinking points, and the introduction of 1,2,3-propanetricarboxylic acid (PTA) gave the LCPs very slight chemical crosslinking. The thermal shape memory and reversible photo-responsive behaviors were investigated, revealing the relationships and interactions between physical and chemical crosslinking. The very slight chemical crosslinking could increase the thermo- and photo-responsive properties of the polyesters but did not worsen the thermoplastic processability.

\section{EXPERIMENTAL SECTION}

\section{Materials}

4-Nitrophenol was manufactured from East China Normal University Chemical Factory (Shanghai, China). 6-Chloro-1-hexanol and 2-phenylsuccinic acid (PSA) were purchased from Aladdin Industrial Corporation (Shanghai, China). 1,2,3-Propanetricarboxylic acid (PTA) was provided by Sinopharm Chemical Reagent Corporation (Chengdu, China). N,N-dimethylformamide (DMF, Ke Long Chemical Co. Chengdu, China) was stored over activated $4 \mathrm{~A}$ molecular sieves before use. All other reagents were commercial and used without further treatment.

\section{Synthesis of PBHPS- $x \%$ PTA}

The monomers, including 4,4'-dihydroxyazobenzene (DHAB) and 4,4'-bis(6-hydroxyhexyloxy)azobenzene (BHHAB) were prepared following the procedure reported in the literature [36,41]. All the polyesters were prepared following the same procedure. The typical polymerization procedure of PBHPS-1\%PTA was as follows: $\mathrm{BHHAB}(4.1454 \mathrm{~g}, 0.01 \mathrm{~mol})$, PSA $(1.9418 \mathrm{~g}$, $0.01 \mathrm{~mol})$ and PTA $(0.0175 \mathrm{~g}, 0.0001 \mathrm{~mol})$ were loaded into a $100-\mathrm{mL}$ three-necked flask, which was equipped with nitrogen inlet and nitrogen outlet, a mechanical stirrer, and a distillation trap connected to a vacuum line. In addition, $0.0122 \mathrm{~g}(0.2 \mathrm{wt} \%) \mathrm{Zn}(\mathrm{Ac})_{2}$ and $0.0183 \mathrm{~g}$ (0.3 wt\%) $\mathrm{Sb}_{2} \mathrm{O}_{3}$ were added into the flask as catalyst. Before the reaction, the flask was processed by vacuum pumping and nitrogen protection to remove the oxygen in it. Then, the flask was placed in a $190^{\circ} \mathrm{C}$ salt bath, the 
esterification reaction proceeded and was kept for about $3 \mathrm{~h}$. After that, a low vacuum was employed and the temperature was preserved at $200^{\circ} \mathrm{C}$ for another $0.5 \mathrm{~h}$. At last, the temperature was kept at $200^{\circ} \mathrm{C}$ with a high vacuum of $20-40 \mathrm{~Pa}$ for about $2.5 \mathrm{~h}$. When the polymerization was completed, the flask was cooled to room temperature under vacuum to obtain the target polymers.

\section{Characterization.}

The swelling ratios of polyesters were measured as follow: all the samples were cut into small slices, swelled and extracted by chloroform for $24 \mathrm{~h}$. The gel content $(G(\%))$ was calculated using the equation:

$$
G(\%)=\frac{m_{1}}{m_{0}} \times 100 \%,
$$

where $m_{1}$ and $m_{0}$ represent the mass of dried extracted sample and original sample, respectively. All the data were averaged over three measurements.

Thermogravimetric analysis (TGA) was conducted under a nitrogen atmosphere using a Netzsch TG 209 F1 instrument. The heating rate and flow rate were kept at $10^{\circ} \mathrm{C} \mathrm{min}^{-1}$ and $60 \mathrm{~mL} \mathrm{~min}^{-1}$ respectively. In each case, a 3-5 mg sample was examined in a temperature range of $40-700^{\circ} \mathrm{C}$.

Thermal transition temperatures of the polyesters were measured using a differential scanning calorimetry (DSC TA Q200 1747) calibrated for both heat flow and temperature using indium and zinc standards. About $5 \mathrm{mg}$ sample was tested under a nitrogen atmosphere at a flow rate of $50 \mathrm{~mL} \mathrm{~min}^{-1}$. Firstly, it was heated to $150^{\circ} \mathrm{C}$ directly for $2 \mathrm{~min}$ to minimize the adverse influence of thermal history. Then the sample was cooled to $-20^{\circ} \mathrm{C}$ and heated to $150^{\circ} \mathrm{C}$ again at a heating rate of $10^{\circ} \mathrm{C} \mathrm{min}^{-1}$. The thermogram was recorded from the first cooling and the second heating cycle.

Liquid crystalline textures of the polyester were observed via a Nikon ECLIPSE LV100 POL polarizing optical microscopy (POM) equipped with a hot-stage. The sample was dissolved in chloroform in a concentration of $10 \mathrm{mg} \mathrm{mL}^{-1}$ and then the solution was cast to a glass flat. After the solvent was evaporated at room temperature, the sample was further dried at $100^{\circ} \mathrm{C}$ for $3 \mathrm{~min}$, and then cooled to $40^{\circ} \mathrm{C}$ at a cooling rate of $10^{\circ} \mathrm{C} \mathrm{min}^{-1}$ for observation.

Wide angle X-ray diffraction (WAXD) was carried out with a Philip X' Pert X-ray diffractometer. The samples were firstly under an isothermal process at $50^{\circ} \mathrm{C}$ for 30 min before test.

Fluorescence spectra were recorded on an F-7000 fluorescence spectrometer (Hitachi, Japan) at the fluorescence emission wavelength of $250 \mathrm{~nm}$. All the samples were dissolved in chloroform to obtain solutions with concentration of $5 \mathrm{mg} \mathrm{mL}^{-1}$ respectively, and then the solution was cast to an optical glass flat. The spectra were recorded after the solvent evaporated at room temperature.

Shape memory behaviors of the polyesters were conducted with dynamic mechanical analysis (DMA, TA Q800). The sample was cut into a rectangular shape with dimensions of approximately $20 \mathrm{~mm} \times 5 \mathrm{~mm} \times 0.5 \mathrm{~mm}$ to test.

UV-vis absorption spectra were recorded on a Varian Cary 50 UV-Vis Spectrophotometer (Varian Co., USA). The photochemical isomerization of the polymer solution was investigated by irradiating it first with a $365 \mathrm{~nm} \mathrm{UV}$ lamp until the photostationary state was reached and then with a $550 \mathrm{~nm}$ visible light lamp. This lamp was also utilized to test the bending and unbending properties of the samples.

The heat-assisted healing process of the scratched polyester films was carried out on the hot stage at $60^{\circ} \mathrm{C}$ and was observed via Nikon ECLIPSE LV100 POL POM. The healing efficiency was tested by tensile test. Two PBHPS-2\%PTA strips of a knife-scratched depth of about $0.1 \mathrm{~mm}$ and an undamaged strip with an average thickness of $0.50 \mathrm{~mm}$ and a width of $3 \mathrm{~mm}$ were prepared. Then, one of the damaged strips was put into an oven for $5 \mathrm{~h}$ at $60^{\circ} \mathrm{C}$. After that, all the strips were tested by INSTORON 3366 Testing Machine under a $100 \mathrm{~N}$ load cell and uniaxially stretched to a displacement at $5 \mathrm{~mm} \mathrm{~min}^{-1}$. The healing efficiencies $(R)$ have been assessed by the equations below [42]. Here $\sigma$ refers to the tensile strength and $\varepsilon$ denotes the elongation at break.

$$
\begin{aligned}
& R(\sigma)=\frac{\sigma_{\text {healed }}}{\sigma_{\text {initial }}} \\
& R(\varepsilon)=\frac{\varepsilon_{\text {healed }}}{\varepsilon_{\text {initial }}}
\end{aligned}
$$

\section{RESULTS AND DISCUSSION}

\section{Polymerization}

The slightly crosslinked liquid crystalline polyesters PBHPS- $x \%$ PTA were synthesized by melting polycondensation. When the temperature reached $190^{\circ} \mathrm{C}$, BHHAB, PSA and PTA melted together into a dilute solution with small bubble. The esterification reaction was kept for about $3 \mathrm{~h}$, and during this time, nitrogen was kept flowing to protect the reaction system from thermal 
Table 1 The swelling test results and TGA data of polyesters

\begin{tabular}{ccccc}
\hline Sample & $G(\%)$ & $T_{5 \%}\left({ }^{\circ} \mathrm{C}\right)$ & $T_{\max }\left({ }^{\circ} \mathrm{C}\right)$ & Residue at $700^{\circ} \mathrm{C}(\%)$ \\
\hline PBHPS & Complete dissolution & 344.0 & 351.6 & 17.8 \\
PBHPS-1\%PTA & $5.9 \pm 2.0$ & 343.7 & 353.6 & 15.8 \\
PBHPS-2\%PTA & $12.6 \pm 0.6$ & 344.2 & 354.9 & 17.0 \\
PBHPS-3\%PTA & $59.4 \pm 9.7$ & 348.0 & 355.7 & 17.5 \\
\hline
\end{tabular}

oxidation and remove the by-product water. As the time went on, the reaction system became viscous and the water could not be taken away by nitrogen, leading the reversible polycondensation to equilibrium. Therefore, the vacuum system was introduced to remove the byproduct and keep the reaction to the positive direction. Then, the polyester became more viscous. For the PBHPS and PBHPS-1\%PTA, the melt adhered to the flask wall finally, but the $2 \%$ and $3 \%$ PTA samples became an elastic substance adhering to the stirring pole. This part of the reaction was kept for about 2 or $3 \mathrm{~h}$ with a high vacuum of 20-40 $\mathrm{Pa}$ according to the different reactive phenomena. Finally, the target product was obtained after cooling the system to room temperature under high vacuum. The swelling test was performed to evaluate the efficiency of the crosslinking, and the results are listed in Table 1. As the contents of PTA increased, the gel contents increased as expected, meaning the density of chemical crosslinking increased.

\section{Thermal properties and liquid crystalline behaviors}

Fig. 1 shows the TGA and DTG curves of the PBHPS$x \%$ PTA samples, and the specific data are summarized in Table 1. All the testing polyesters had one single degradation process. The temperatures of $5 \mathrm{wt} \%$ loss $\left(T_{5 \%}\right.$, defined as the initial decomposition temperature) and $T_{\max }$ were nearly the same, indicating that the incorporation of PTA caused a slight chemical crosslinking

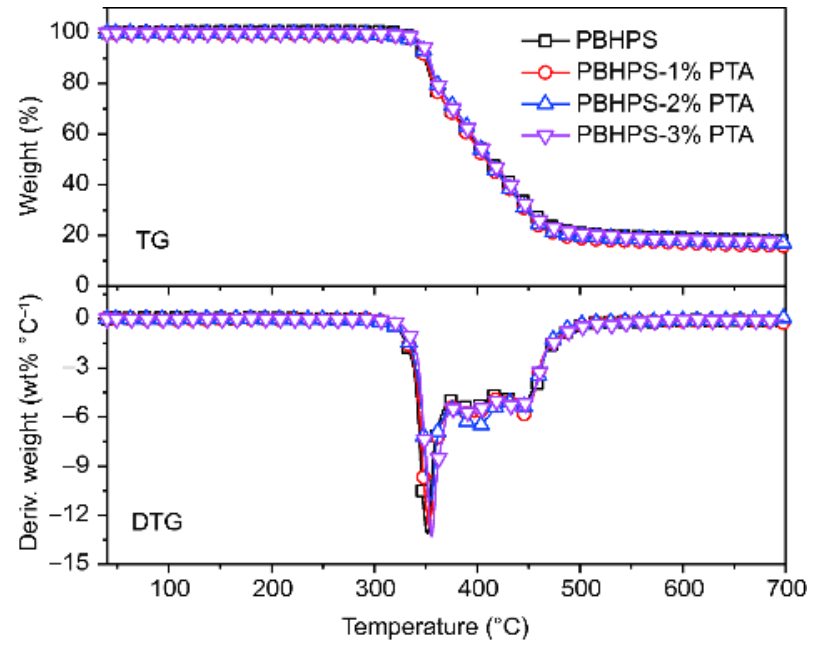

Figure 1 TGA and DTG curves of liquid crystalline polyesters in $\mathrm{N}_{2}$.

but did not affect the thermal stability of the polyesters.

Fig. 2 illustrates the DSC curves of the PBHPS- $x \%$ PTA samples in the first cooling scan (a) and the second heating scan (b) at a rate of $10^{\circ} \mathrm{C} \mathrm{min}^{-1}$ under $\mathrm{N}_{2}$ atmosphere. The relevant thermal parameters and their corresponding values are summarized in Table 2 . The samples, except PBHPS-3\%PTA, had nearly the same glass transition temperatures $\left(T_{\mathrm{g}}\right)$ and isotropic temperatures $\left(T_{\mathrm{i}}\right)$ at about $22^{\circ} \mathrm{C}$ and $70^{\circ} \mathrm{C}$ in the first cooling process, which meant that the incorporation of low
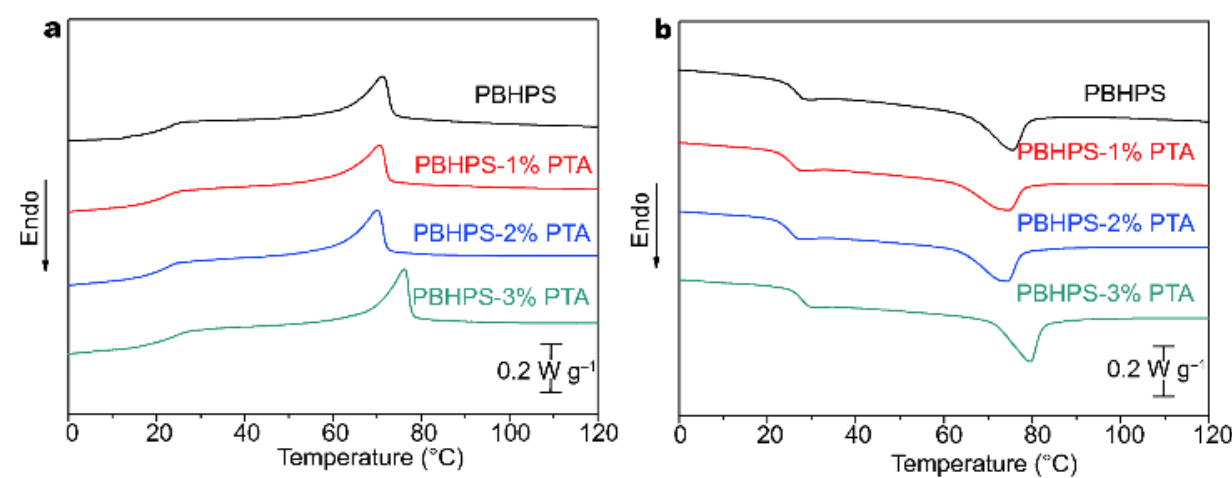

Figure 2 DSC curves of the polyesters on (a) first cooling and (b) second heating at a rate of $10^{\circ} \mathrm{C} \min ^{-1}$ under $\mathrm{N}_{2}$ atmosphere. 
Table 2 DSC data of the polyesters at a rate of $10^{\circ} \mathrm{C} \mathrm{min}{ }^{-1}$ under $\mathrm{N}_{2}$ atmosphere

\begin{tabular}{|c|c|c|c|c|c|c|}
\hline \multirow{2}{*}{ Sample } & \multicolumn{3}{|c|}{ First cooling } & \multicolumn{3}{|c|}{ Second heating } \\
\hline & $T_{\mathrm{g}}\left({ }^{\circ} \mathrm{C}\right)$ & $T_{\mathrm{i}}\left({ }^{\circ} \mathrm{C}\right)$ & $\Delta H_{\mathrm{i}}\left(\mathrm{J} \mathrm{g}^{-1}\right)$ & $T_{\mathrm{g}}\left({ }^{\circ} \mathrm{C}\right)$ & $T_{\mathrm{i}}\left({ }^{\circ} \mathrm{C}\right)$ & $\Delta H_{\mathrm{i}}\left(\mathrm{J} \mathrm{g}^{-1}\right)$ \\
\hline PBHPS & 22.3 & 71.5 & 7.1 & 26.9 & 75.8 & 7.0 \\
\hline PBHPS-1\%PTA & 21.9 & 70.5 & 7.6 & 25.6 & 73.8 & 7.6 \\
\hline PBHPS-2\%PTA & 22.6 & 70.0 & 8.0 & 25.2 & 73.3 & 7.9 \\
\hline PBHPS-3\%PTA & 24.6 & 76.2 & 8.4 & 27.7 & 79.2 & 8.4 \\
\hline
\end{tabular}

contents of PTA did not influence the phase transition temperatures. However, when the content of PTA increased to $3 \%$, both $T_{\mathrm{g}}$ and $T_{\mathrm{i}}$ slightly increased to $24.6^{\circ} \mathrm{C}$ and $76.2^{\circ} \mathrm{C}$, respectively. This was reasonable because the relative high density of crosslinking would district the movement of the polymer chains, thus increasing the relevant temperatures.

It was noteworthy that all the polyesters with PTA crosslinking still showed the isotropic temperatures, meaning that the crosslinking did not negatively affect the melting processability. For further confirming the thermoplasticity of these polyesters, PBHPS-2\%PTA and PBHPS-3\%PTA samples with higher crosslinking density were chosen to undergo a 5 time cooling and heating cycles by using DSC respectively. As shown in Fig. 3, during the multiple cooling and heating process, the tested samples exhibited the $T_{\mathrm{g}}$ and $T_{\mathrm{i}}$, and these phase transition temperatures were kept the same all the time, which further confirmed the slight crosslinking did not influence the thermoplasticity of the resulting polyesters.
Fig. 4 shows the liquid crystalline textures of PBHPS and PBHPS- $x \% \mathrm{PTA}$ on the first cooling process, which were observed and captured at $40^{\circ} \mathrm{C}$ by using a POM. All the slightly crosslinking samples showed the typical focal conic textures as PBHPS did [26], which implied the small quantity of PTA incorporated into the PBHPS chains did not change the molecular mobility and regular packing, as illustrated in DSC test. WAXD analysis was also used to determine the microstructure of the polyesters. As shown in Fig. 5a, in the small angle region, except PBHPS-3\%PTA, there were two distinct peaks at $6.3^{\circ}(1.39 \mathrm{~nm})$ and $12.5^{\circ}(0.71 \mathrm{~nm})$, suggesting the existence of the layer structure in the liquid crystalline chain. For PBHPS-3\%PTA, the peaks in the small angle range were negligible, because the relative high content of PTA influenced the chain folding or arrangement, thus destroyed the orderly structure between the layers. For PBHPS, there was a peak at the angle of $28.1^{\circ}(0.32 \mathrm{~nm})$ which indicated the $\pi-\pi$ interactions between the phenyl rings exiting in the polymer chains as reported previously
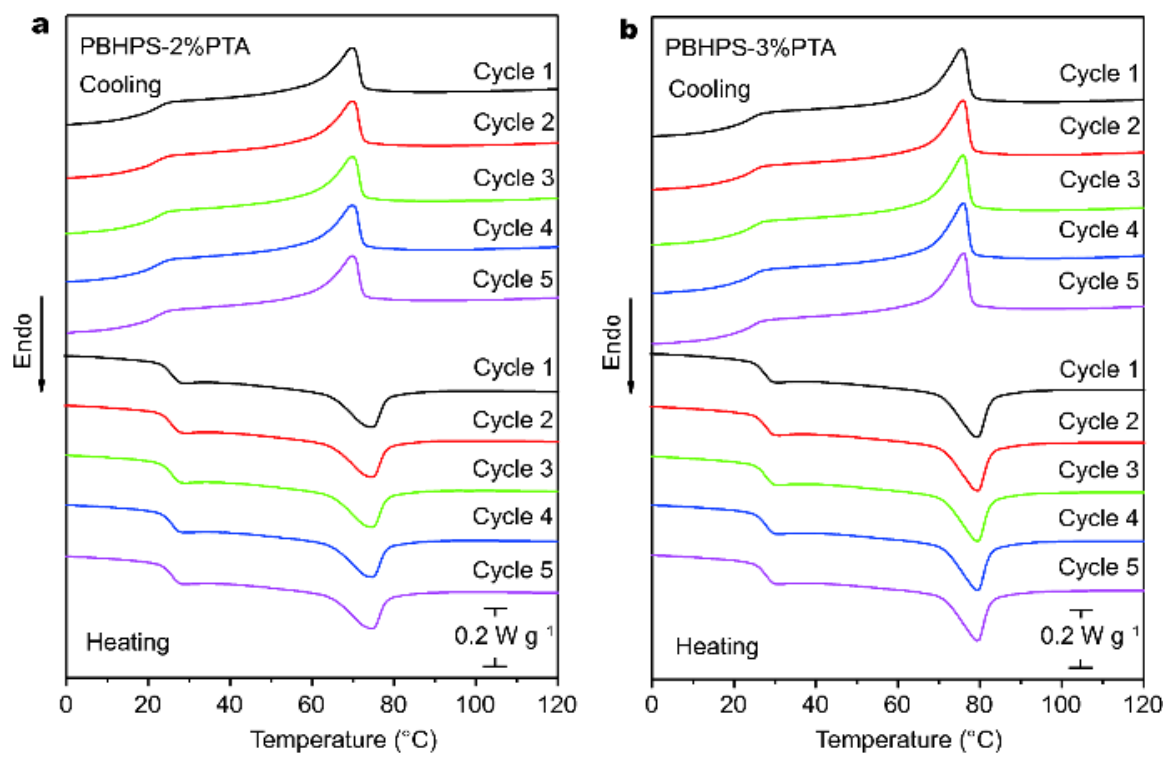

Figure 3 DSC curves of PBHPS-2\%PTA (a) and PBHPS-3\%PTA (b) in five cooling and heating cycles at a rate of $10^{\circ} \mathrm{C}$ min $^{-1}$ under $\mathrm{N}_{2}$ atmosphere. 


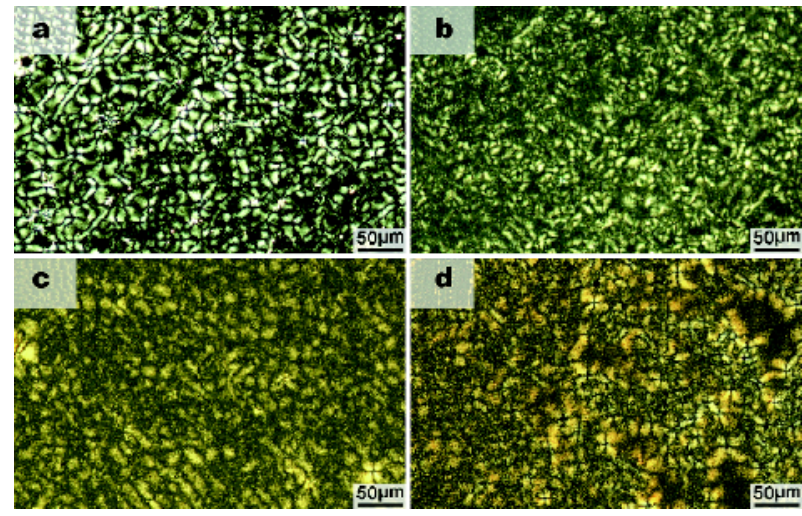

Figure 4 POM pictures of PBHPS (a), PBHPS-1\%PTA (b), PBHPS-2\% PTA (c) and PBHPS-3\%PTA (d) at $40^{\circ} \mathrm{C}$.

$[23,26]$. When $1 \%$ of PTA was incorporated, peaks in this area still existed, impling the $\pi-\pi$ interactions remained in PBHPS-1\%PTA. However, the peaks at about $28^{\circ}$ for the polyesters PBHPS-2\%PTA and PBHPS-3\%PTA decreased obviously, which meant the $\pi-\pi$ interactions might be weakened or broken. To further reveal the relation between the PTA (chemical crosslinking) and the $\pi-\pi$ interactions (physical crosslinking), fluorescence spectra test was performed to study the intensity of $\pi-\pi$ interactions-the fluorescence emission means there exists conjugation in the system $[35,43]$. As shown in Fig. $5 \mathrm{~b}$, the intensity of the fluorescence emission spectra decreased with increasing the contents of PTA, meaning the $\pi-\pi$ interactions were decreased even disappeared gradually. This process could suggest that the physical crosslinking was replaced by the chemical crosslinking slowly but surely.

Thermally induced shape memory properties In this part, the thermally induced shape memory prop- erties of the polyesters were characterized by a well-established four-step thermo-mechanical cycling method conducted via DMA. All the samples were stretched at $40^{\circ} \mathrm{C}$ firstly and then the strain was ramped to about $50 \%$ gradually (deformation). After that a rapid cooling procedure was executed, during which the force was kept constant until the temperature reached $10^{\circ} \mathrm{C}$. In the third step, the stress was gradually released to witness the strain fixing at $10^{\circ} \mathrm{C}$. In the fourth step, the strain recovery occurred by heating to $60^{\circ} \mathrm{C}$ at the rate of $10^{\circ} \mathrm{C} \mathrm{min}^{-1}$ (recovery). According to the Equations (4) and (5), where $\varepsilon_{\mathrm{m}}, \varepsilon_{\mu}, \varepsilon_{\mathrm{p}}$ and $N$ represent the strain before unloading, the strain after unloading, the permanent (residual) strain after heat induced recovery and the number of cycles, respectively, the fixing ration $\left(R_{\mathrm{f}}\right)$ and the recovery ratio $\left(R_{\mathrm{r}}\right)$ were calculated to assess the shape memory property.

$$
\begin{array}{r}
R_{\mathrm{f}}=\frac{\varepsilon_{\mu}(N)-\varepsilon_{\mathrm{p}}(N-1)}{\varepsilon_{\mathrm{m}}(N)-\varepsilon_{\mathrm{p}}(N-1)} \times 100 \% \\
R_{\mathrm{r}}=\frac{\varepsilon_{\mu}(N)-\varepsilon_{\mathrm{p}}(N)}{\varepsilon_{\mu}(N)-\varepsilon_{\mathrm{p}}(N-1)} \times 100 \%
\end{array}
$$

Typically, Fig. 6e presents the thermally induced shape memory results of PBHPS-2\%PTA sample investigated by cyclic thermomechanical analysis, while those of others were provided in Fig. S1. The corresponding data are summarized in Table 3. Generally, the results recorded within the first cycle were strongly dependent on the residual stresses of samples resulting from processing history. The $R_{\mathrm{f}}$ and $R_{\mathrm{r}}$ were therefore calculated from the data determined within the $2^{\text {nd }}$ to the $4^{\text {th }}$ cycle. All the samples had good shape fixing ratios about $98 \%$ : as the temperature came to $10^{\circ} \mathrm{C}$, all the polyesters were at glassy state and lost their chain mobility, and the storing elastic energy was frozen at the same time. However, the recovery ratios were significantly different with different
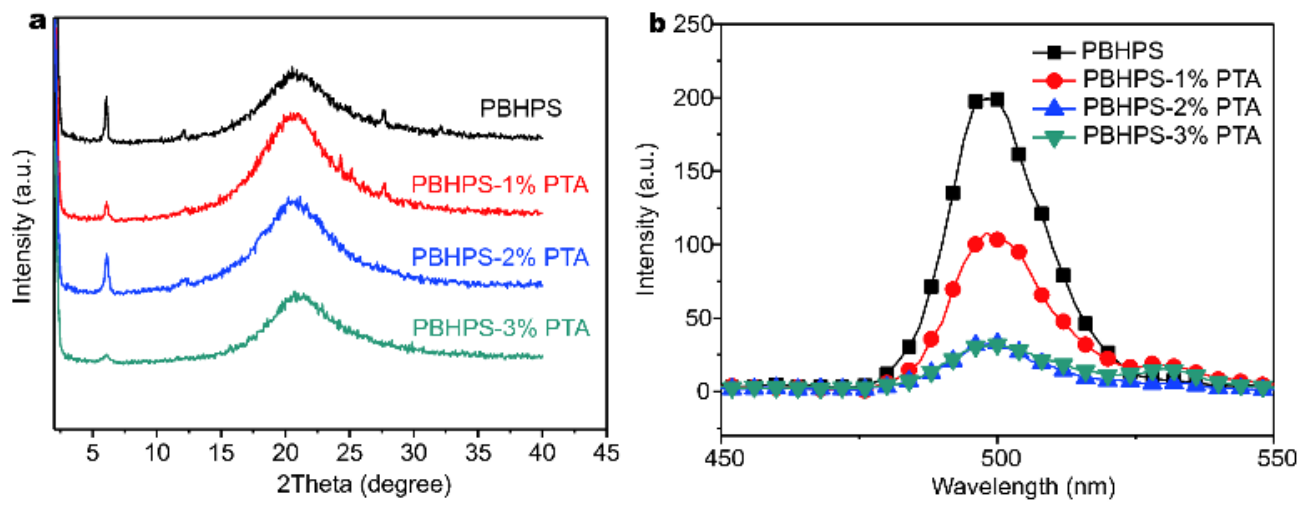

Figure 5 XRD patterns of the polyesters at room temperature (a). The intensity of the fluorescence emission spectra (b) of the polyesters. 

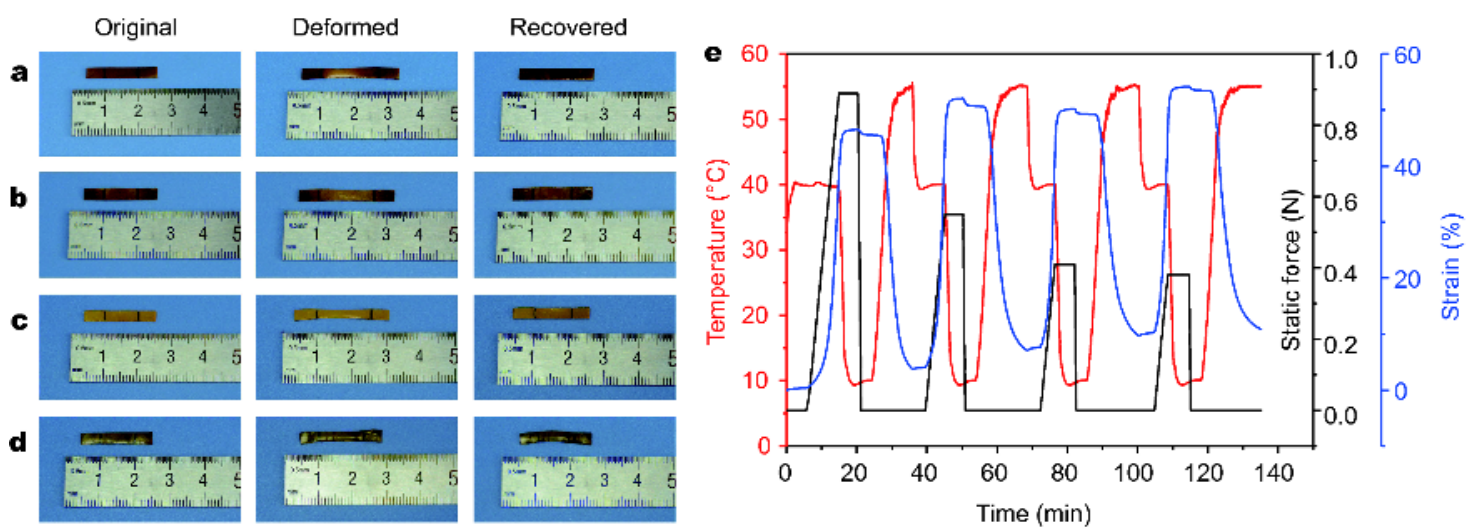

Figure 6 Digital photograph of shape memory process of polyesters: (a) PBHPS, (b) PBHPS-1\% PTA, (c) PBHPS-2\% PTA and (d) PBHPS-3\% PTA. Programmed cyclic thermomechanical curves of PBHPS-2\%PTA obtained by DMA (e).

Table 3 Fixing and recovery ratio of the polyesters obtained by DMA

\begin{tabular}{ccc}
\hline Sample & Fixing ratio (\%) & Recovery ratio (\%) \\
\hline PBHPS & $98.6 \pm 0.4$ & $94.4 \pm 0.9$ \\
PBHPS-1\%PTA & $97.9 \pm 0.2$ & $85.3 \pm 1.1$ \\
PBHPS-2\%PTA & $97.9 \pm 0.4$ & $94.0 \pm 2.6$ \\
PBHPS-3\%PTA & $99.5 \pm 0.1$ & $98.6 \pm 0.4$ \\
\hline
\end{tabular}

content of PTA. When the content of PTA was $1 \%$, the recovery ratio of $\mathrm{PBHPS}-1 \% \mathrm{PTA}$ dropped obviously to about $85.3 \%$. As the introduction of PTA, the chemical crosslinking strongly influenced the chain conformation. As a consequence, some physical crosslinking $(\pi-\pi$ interactions) did not form effectively, and the intensity of physical crosslinking became weak. However, when the PTA content was relatively low, the introduced chemical crosslinking was not strong enough to force the deformed sample to recover, resulting in a sharply decline of the recovery ratio. As the amount of PTA increased sequentially, the recovery ratio of the polyesters rose again. The recovery ratio of PBHPS-3\%PTA reached 98.6\%. With relative high content of PTA, the chemical crosslinking became more dominant than the physical crosslinking in the system, thus making the shape memory properties much better. Fig. 6 shows digital photographs of the recovery process of these polyesters. The samples were firstly stretched and then recovered in the hot stage at $60^{\circ} \mathrm{C}$. The results of all these polyesters' shape memory properties were in accordance with the DMA tests.

\section{Photo-responsive behavior}

The photo-responsive properties of all these polyesters were investigated by UV-vis spectroscopy in DMF solu- tion under irradiation of $365 \mathrm{~nm}$ light and $550 \mathrm{~nm}$ light, as illustrated in Fig. 7 (PBHPS-2\%PTA). When irradiated at $365 \mathrm{~nm}$, the solution underwent trans to cis photoisomerization until a photostationary state was eventually reached. The intensity of the $\pi-\pi^{*}$ transition band around $360 \mathrm{~nm}$ decreased with the irradiation time, whereas that of the $n-\pi^{*}$ transition band around $450 \mathrm{~nm}$ slightly increased. Then the cis to trans back-isomerization process happened when the samples were irradiated at visible light $(\lambda=550 \mathrm{~nm})$. The $\pi-\pi^{*}$ transition band increased and the $n-\pi^{*}$ transition band decreased back. However, the finally recovered absorbance of the trans-isomer was lower than that before UV irradiation. This phenomenon was reported before [31] but the reason was not clear yet. One possible explanation is that when the cis to trans photochemical back-isomerization was performed, there was also a weak absorption from the trans-isomer at the same wavelength, which led to an equilibrium between these two isomers during the isomerization process under the same visible light after most of the cis-isomer returned to the trans-isomer. The other three polyesters displayed the similar properties under the irradiation of UV light and visible light, and the results were given in the supporting information (Fig. S2).

The macroscopical photo-mechanical properties of all these polyesters were then studied. The simple melt stretching method was used to get the vimineous samples with a relatively high order of mesogen along the stretch direction for the test [31,37]. Fig. 8 shows the photoinduced bending and unbending behaviors of these polyesters upon irradiation with UV light and visible light, respectively. It was seen clearly that the PBHPS bent to the UV light source direction when it was exposed to $365 \mathrm{~nm}$ UV light $\left(15 \mathrm{~mW} \mathrm{~cm}^{-2}\right)$ from above of the sam- 

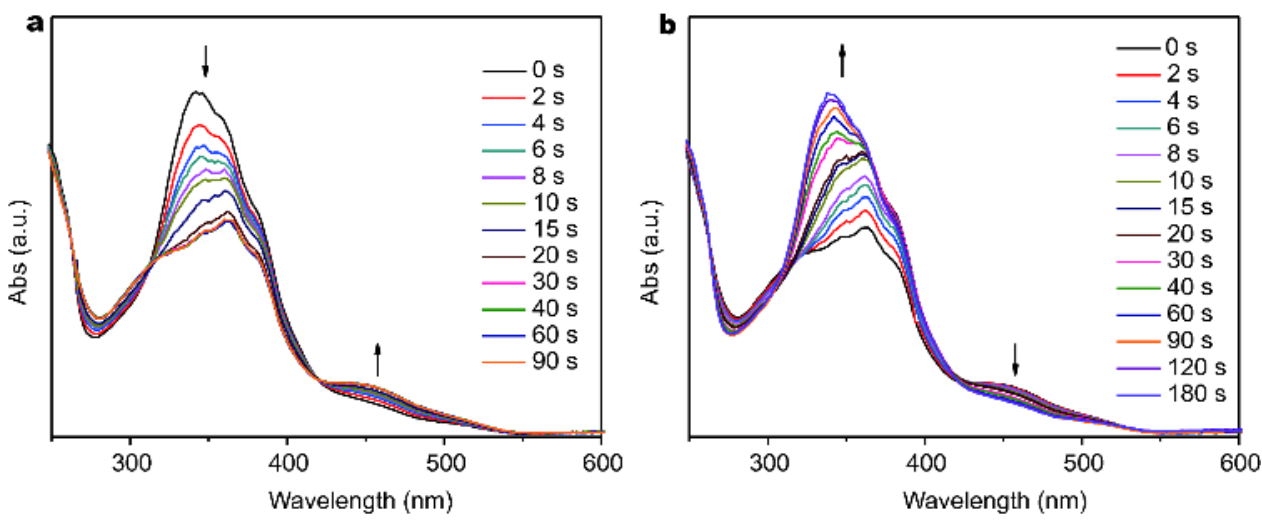

Figure 7 UV-vis spectral changes with time for the solution of PBHPS-2\%PTA in DMF at room temperature upon irradiation with $365 \mathrm{~nm}$ UV light (a) and upon irradiating the polymer solution at the photostationary state with $550 \mathrm{~nm}$ visible light (b).

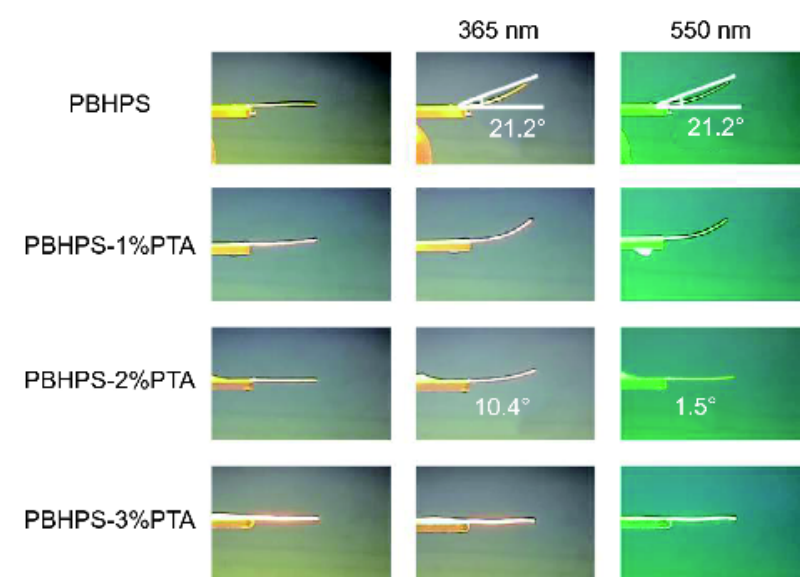

Figure 8 Digital pictures of the polyester films motion in the presence of UV light $\left(365 \mathrm{~nm}, 15 \mathrm{~mW} \mathrm{~cm}^{-2}\right)$ and visible light $(550 \mathrm{~nm}$, $15 \mathrm{~mW} \mathrm{~cm}^{-2}$ ).

ple. However, the PBHPS sample did not revert to its initial straight state upon irradiation with visible light $\left(550 \mathrm{~nm}, 15 \mathrm{~mW} \mathrm{~cm}^{-2}\right)$. This was because the aromatic structure in the PBHPS chain slipped during the isomerization process of the azobenzene groups under the UV light, resulting in losing the physical crosslinking points in the system. As the bending process finished, a kind of new $\pi-\pi$ stacking formed, fixing the deformed bending shape. When irradiated by the visible light, the newly formed $\pi-\pi$ stacking was broken again as the cis to trans isomerization happened. However, the final alignment of trans azobenzene mesogens were different from the original ones in the absent of some hard segment to restrict the movement of the polymer chain. Thus, the PBHPS sample could not recover to its original straight state. For the sample PBHPS-1\%PTA, the introduction of $1 \%$ PTA did not afford enough chemical crosslinking as a

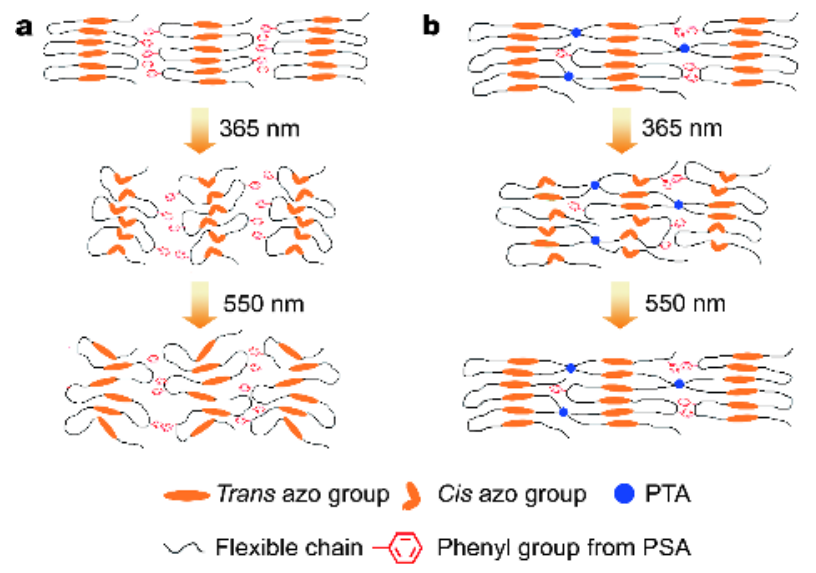

Figure 9 The schematic drawing of the oriented samples PBHPS (a) and PBHPS-PTA (b) during the isomerization process under the UV light and visible light.

hard segment, and thus the photo-mechanical property of PBHPS-1\%PTA was similar to that of PBHPS. A schematic drawing of this process is shown in Fig. 9a. When more PTA was incorporated to form chemical crosslinking, the reversible photo-induced bending and unbending properties were improved. Also shown in Fig. 8, the PBHPS-2\%PTA samples could bend under the UV light irradiation, and revert to their straight state upon the irradiation with the $550 \mathrm{~nm}$ visible light with a recovery ratio of $85.6 \%$, although the bending angles were decreased, which could be attributed to the chemical crosslinking in the polymer chains. Since the chemical crosslinking did not response to the UV light and restricted the mobility of the azobenzene groups, these crosslinking points (fixing phase) remained during the isomerization of azobenzene groups under UV light, and then forced the polymer chains back to the initial align- 

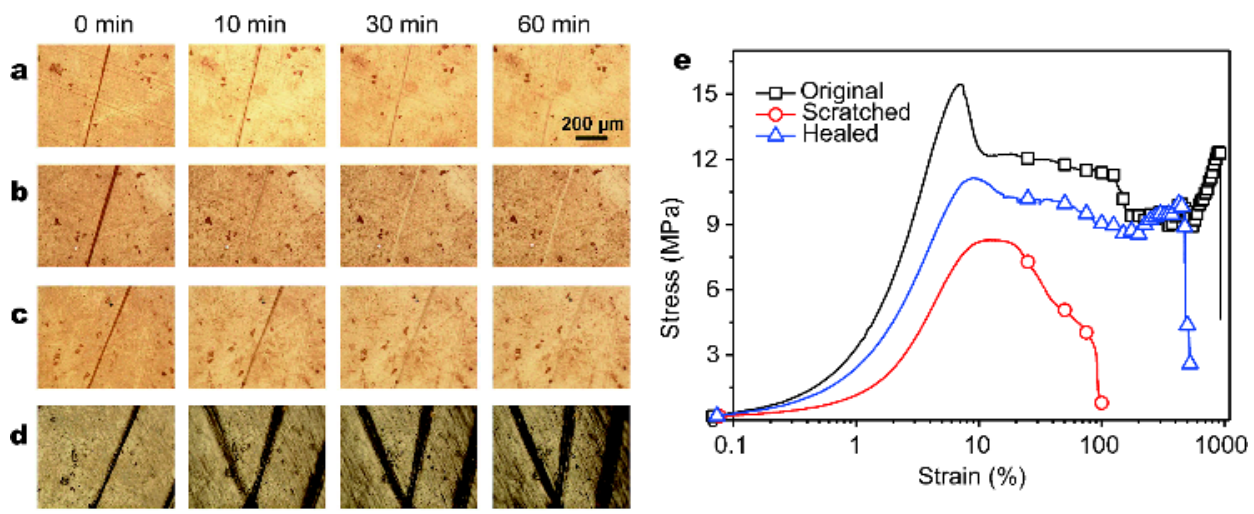

Figure 10 Self-healing process of the scratched polyesters observed by POM at $60^{\circ} \mathrm{C}$ : (a) PBHPS, (b) PBHPS-1\%PTA, (c) PBHPS-2\%PTA and (d) PBHPS-3\%PTA. Stress versus strain curves for the original, scratched and healed state of PBHPS-2\%PTA (e).

ment under the visible light. The schematic drawing of this process is shown in Fig. 9b. However, as the PTA chemical crosslinking became dominant in the polymer chains, the mechanical properties were enhanced (Fig. S3) and the movement of the polymer chains were restricted gradually, causing the bending angles of the crosslinked polyesters to decrease obviously. Even the PBHPS$3 \%$ PTA sample could not bend when induced by the UV light.

\section{Heat-assisted healing behavior}

In our previous work [36,37], the azobenzene based main-chain liquid crystalline polyester PBHPS showed heat-assisted healing behavior due to the $\pi-\pi$ interaction in the chain as the physical crosslink. When crosslinker PTA is introducd into PBHPS to form some slight chemical crosslink, would the chemical crosslink influence the healing behaviors? Firstly, the scratch healing behaviors of the polyesters were studied by using POM. The scratched polyester films were put on the $60^{\circ} \mathrm{C}$ hot stage and the heat-assisted healing process was recorded by the digital camera equipped on the POM. The snapshots were captured at different time. As shown in Fig. 10, the PBHPS-1\%PTA sample with lower content of PTA showed similar healing behavior as PBHPS because of the relatively strong $\pi-\pi$ interaction in the polymer chains. When the healing process occurred at $60^{\circ} \mathrm{C}$, the polyesters were in liquid crystalline phase, making the $\pi-\pi$ stacking reformed at the presence of the position order of the mesogenic units. When the content of PTA increased, some $\pi-\pi$ stacking physical crosslink did not form effectively which was proved by WXAD and fluorescence emission spectra above. Thus, the heat-assisted healing properties of PBHPS-2\%PTA and PBHPS-3\%PTA decreased. For PBHPS-2\%PTA, there still existed a blurred scratch after healing $60 \mathrm{~min}$. The PBHPS-3\%PTA shrank did not heal at the temperature of $60^{\circ} \mathrm{C}$, proving the $\pi-\pi$ interaction played an important role in heat-assisted healing behavior indirectly.

Herein, the PBHPS-2\%PTA was chosen to study the healing efficiency. The healing efficiency of scratched healing method was measured by tensile test, as shown in Fig. 10e. The tensile stress and the elongation at break of the original PBHPS-2\%PTA was $15.5 \mathrm{MPa}$ and $928.9 \%$, respectively; and the scratched one decreased to $8.3 \mathrm{MPa}$ and $107.9 \%$, where the testing sample fractured precisely at the scratch trace. After $5 \mathrm{~h}$ healing process at $60^{\circ} \mathrm{C}$ and without the compression stress, the tensile stress of the healed one recovered to $11.1 \mathrm{MPa}$ and $529.8 \%$, thus the healing efficiency was $71.6 \%$ (for strength) and $57.0 \%$ (for elongation), respectively.

\section{CONCLUSION}

In conclusion, a series of azobenzene-containing liquid crystalline polyesters combining physical and chemical crosslinking were synthesized successfully. All of these polyesters showed good thermal stability and their thermal decomposition temperature was higher than $340^{\circ} \mathrm{C}$. DSC showed that all these crosslinked samples had a relative low phase transition temperatures with thermoplastic processability. With POM and XRD results, these polyesters showed smectic liquid crystalline phase. Furthermore, there also exist strong $\pi-\pi$ stacking interactions in the polymer chains with low content of PTA as the crosslinking agent. When PTA content increased, as illustrated in XRD and fluorescence emission spectra, the chemical crosslinking negatively influenced the original physical crosslinking ( $\pi-\pi$ interaction) among the polymer chains. The thermally induced shape memory behaviors of these polyesters were evaluated by DMA: all 
these samples exhibited expected fixing ratios about 98\%; however, the recovery ratios changed obviously with different content of PTA. The chemical crosslinking also influenced the photo responsive performance of these polyesters. At the absence of chemical crosslinking, the oriented sample (PBHPS) could be bent under the irradiation of UV light; however, since the isomerization process of azobenzene groups broke the $\pi-\pi$ interactions in the polymer chains, the bent sample could not revert straight under the irradiation of visible light. At the presence of slight chemical crosslinking, thanks to the inert response of the crosslinking to the UV light, such problem was solved by restricting the mobility of the azobenzene groups, then forcing the polymer chains back to the initial alignments under the visible light. As a consequence, the reversible bending and unbending behaviors of the slightly crosslinked polyesters were achieved. The heat-assisted healing properties of these polyesters were achieved as the physical crosslinking dominated in the chains. Therefore, this main-chain azobenzene-containing liquid crystalline polyester could be utilized as a kind of smart polymer and exhibits its own value in the industry field.

\section{Received 23 January 2018; accepted 9 March 2018; published online 27 March 2018}

1 Warner M, Terentjev E. Liquid Crystal Elastomers. Oxford: Oxford University Press, 2003

2 de Jeu WH. Liquid Crystal Elastomers: Materials and Applications. Heidelberg : Springer, 2012, pp. V-VI

3 Huang WM, Zhao Y, Wang CC, et al. Thermo/chemo-responsive shape memory effect in polymers: a sketch of working mechanisms, fundamentals and optimization. J Polym Res, 2012, 19: 9952

$4 \mathrm{Ohm} \mathrm{C}$, Brehmer M, Zentel R. Liquid crystalline elastomers as actuators and sensors. Adv Mater, 2010, 22: 3366-3387

5 Degennes PG. One type of nematic polymers. C R Hebd Sean Acad Sci Ser B, 1975, 281: 101-103

6 Burke KA, Rousseau IA, Mather PT. Reversible actuation in mainchain liquid crystalline elastomers with varying crosslink densities. Polymer, 2014, 55: 5897-5907

7 García-Márquez AR, Heinrich B, Beyer N, et al. Mesomorphism and shape-memory behavior of main-chain liquid-crystalline coelastomers: modulation by the chemical composition. Macromolecules, 2014, 47: 5198-5210

8 Ahn S, Deshmukh P, Gopinadhan M, et al. Side-chain liquid crystalline polymer networks: exploiting nanoscale smectic polymorphism to design shape-memory polymers. ACS Nano, 2011, 5: 3085-3095

9 Ahn S, Kasi RM. Exploiting microphase-separated morphologies of side-chain liquid crystalline polymer networks for triple shape memory properties. Adv Funct Mater, 2011, 21: 4543-4549

10 Burke KA, Mather PT. Crosslinkable liquid crystalline copolymers with variable isotropization temperature. J Mater Chem, 2012, 22: 14518-14530
11 Wen Z, Zhang T, Hui Y, et al. Elaborate fabrication of well-defined side-chain liquid crystalline polyurethane networks with tripleshape memory capacity. J Mater Chem A, 2015, 3: 13435-13444

12 Finkelmann H, Nishikawa E, Pereira GG, et al. A new opto-mechanical effect in solids. Phys Rev Lett, 2001, 87: 015501

13 Yu Y, Nakano M, Ikeda T. Directed bending of a polymer film by light. Nature, 2003, 425: 145-145

14 van Oosten CL, Bastiaansen CWM, Broer DJ. Printed artificial cilia from liquid-crystal network actuators modularly driven by light. Nat Mater, 2009, 8: 677-682

15 Cheng F, Yin R, Zhang Y, et al. Fully plastic microrobots which manipulate objects using only visible light. Soft Matter, 2010, 6: 3447-3449

16 Lee KM, Smith ML, Koerner H, et al. Photodriven, flexural-torsional oscillation of glassy azobenzene liquid crystal polymer networks. Adv Funct Mater, 2011, 21: 2913-2918

17 Ware TH, McConney ME, Wie JJ, et al. Voxelated liquid crystal elastomers. Science, 2015, 347: 982-984

18 Sun L, Huang WM, Ding Z, et al. Stimulus-responsive shape memory materials: A review. Mater Des, 2012, 33: 577-640

19 Liu C, Qin H, Mather PT. Review of progress in shape-memory polymers. J Mater Chem, 2007, 17: 1543-1558

20 Zhao Q, Qi HJ, Xie T. Recent progress in shape memory polymer: New behavior, enabling materials, and mechanistic understanding. Prog Polymer Sci, 2015, 49-50: 79-120

21 Salvekar AV, Zhou Y, Huang WM, et al. Shape/temperature memory phenomena in un-crosslinked poly--caprolactone (PCL). Eur Polymer J, 2015, 72: 282-295

22 Küpfer J, Finkelmann H. Macromol Chem Rapid Commun, 1991, 12: $717-726$

23 Broer DJ, Boven J, Mol GN, et al. In stiu photopolymerization of oriented liquid-crystalline acrylates 3 . Oriented polymer networks from a mesogenic diacrylate. Macromol Chem Phys, 1989, 190: 2255-2268

24 Pei Z, Yang Y, Chen Q, et al. Mouldable liquid-crystalline elastomer actuators with exchangeable covalent bonds. Nat Mater, 2014, 13: $36-41$

25 Li Y, Rios O, Keum JK, et al. Photoresponsive liquid crystalline epoxy networks with shape memory behavior and dynamic ester bonds. ACS Appl Mater Interfaces, 2016, 8: 15750-15757

26 Michal BT, McKenzie BM, Felder SE, et al. Metallo-, thermo-, and photoresponsive shape memory and actuating liquid crystalline elastomers. Macromolecules, 2015, 48: 3239-3246

27 Qin C, Feng Y, Luo W, et al. A supramolecular assembly of crosslinked azobenzene/polymers for a high-performance light-driven actuator. J Mater Chem A, 2015, 3: 16453-16460

28 Lv JA, Liu Y, Wei J, et al. Photocontrol of fluid slugs in liquid crystal polymer microactuators. Nature, 2016, 537: 179-184

29 Lv J, Wang W, Wu W, et al. A reactive azobenzene liquid-crystalline block copolymer as a promising material for practical application of light-driven soft actuators. J Mater Chem C, 2015, 3: 6621-6626

30 Mamiya J, Yoshitake A, Kondo M, et al. Is chemical crosslinking necessary for the photoinduced bending of polymer films? J Mater Chem, 2008, 18: 63-65

31 Fang L, Zhang H, Li Z, et al. Synthesis of reactive azobenzene main-chain liquid crystalline polymers via michael addition polymerization and photomechanical effects of their supramolecular hydrogen-bonded fibers. Macromolecules, 2013, 46: 7650-7660

32 Zhao R, Zhao T, Jiang X, et al. Thermoplastic high strain multi- 
shape memory polymer: side-chain polynorbornene with columnar liquid crystalline phase. Adv Mater, 2017, 29: 1605908

33 Yang R, Chen L, Ruan C, et al. Chain folding in main-chain liquid crystalline polyesters: from $\pi-\pi$ stacking toward shape memory. J Mater Chem C, 2014, 2: 6155-6164

34 Meng ZY, Chen L, Zhong HY, et al. The effect of carbon nanotube plus graphene on the shape memory behavior and tensile properties of a liquid crystalline polyester. Acta Polym Sin, 2016, 12: 1758-1762

35 Meng ZY, Chen L, Zhong HY, et al. Effect of different dimensional carbon nanoparticles on the shape memory behavior of thermotropic liquid crystalline polymer. Composites Sci Tech, 2017, 138: 8-14

36 Zhong HY, Chen L, Yang R, et al. Azobenzene-containing liquid crystalline polyester with $\pi-\pi$ interactions: diverse thermo- and photo-responsive behaviours. J Mater Chem C, 2017, 5: 3306-3314

37 Zhong HY, Chen L, Liu XF, et al. Novel liquid crystalline copolyester containing amphi-mesogenic units toward multiple stimuliresponse behaviors. J Mater Chem C, 2017, 5: 9702-9711

38 Yang R, Ding L, Chen W, et al. Chain folding in main-chain liquid crystalline polyester with strong $\pi-\pi$ interaction: An efficient $\beta$ nucleating agent for isotactic polypropylene. Macromolecules, 2017, 50: 1610-1617

39 Montarnal D, Tournilhac F, Hidalgo M, et al. Epoxy-based networks combining chemical and supramolecular hydrogen-bonding crosslinks. J Polym Sci A Polym Chem, 2010, 48: 1133-1141

40 Pan Y, Liu T, Li J, et al. High modulus ratio shape-memory polymers achieved by combining hydrogen bonding with controlled crosslinking. J Polym Sci B Polym Phys, 2011, 49: 12411245
41 Chakraborty S, Rajput L, Desiraju GR. Designing ternary cocrystals with stacking interactions and weak hydrogen bonds. 4,4'bis-hydroxyazobenzene. Cryst Growth Des, 2014, 14: 2571-2577

42 Wool RP, O'Connor KM. A theory crack healing in polymers. J Appl Phys, 1981, 52: 5953-5963

43 Ahn J, Park S, Lee JH, et al. Fluorescent hydrogels formed by $\mathrm{CH}-$ $\pi$ and $\pi-\pi$ interactions as the main driving forces: an approach toward understanding the relationship between fluorescence and structure. Chem Commun, 2013, 49: 2109-2111

Acknowledgements This work was supported by the National Natural Science Foundation of China (51721091) and the Sichuan Province Youth Science and Technology Innovation Team (2017TD0006). The authors would also like to thank the Analysis and Testing Center of Sichuan University for the NMR measurement.

Author contributions Zhong HY and Ding XM carried out the experiments; Zhong HY and Chen L analyzed the data and wrote the draft of manuscript; Chen L and Wang YZ proposed the project and critical comments on the writing of the manuscript; Yang $\mathrm{R}$ provided some additional suggestions and comments on analyzing the data. All the authors checked and approved the manuscript.

Conflict of interest The authors declare that they have no conflict of interest.

Supplementary information Shape memory tests of polyesters by DMA, UV-vis spectra of PBHPS, PBHPS-1\%PTA and PBHPS-3\%PTA solution in DMF and the storage modulus of these polymers tested by DMA are available in the online version of the paper. 

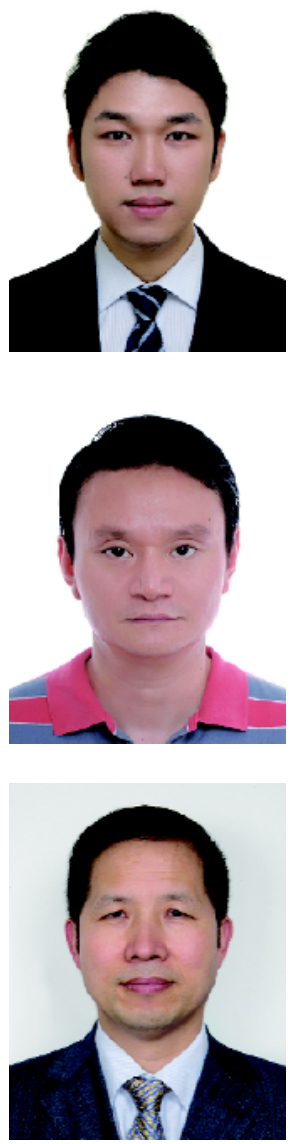

Hi-Yi Zhong earned his PhD degree in Polymer Chemistry and Physics (2017) and BSc degree in Chemistry (2012) from Sichuan University under the supervision of Prof. Yu-Zhong Wang. His research interest is the stimuli responsive liquid crystalline polymers. He is currently a lecturer in the College of Pharmacy, Guangxi University of Chinese Medicine.

Li Chen is currently a full professor in the College of Chemistry, Sichuan University. He earned his PhD degree in Polymer Chemistry and Physics (2009) and MSc degree in Materials Science (2006) from Sichuan University, and BSc degree in Chemistry (2003) from Hunan University. In 2009, he joined Professor Yu-Zhong Wang's group and his current research interests are focused on the synthesis, structure and properties of functional liquid crystalline polymers and flame-retardant materials.

Yu-Zhong Wang earned his PhD degree from Sichuan University in 1994, where he was promoted to a full Professor in 1995. He is the Director of the National Engineering Laboratory for Eco-Friendly Polymeric Materials (Sichuan). His research interests are focused on fire-retardant and functional polymeric materials, bio-based and biodegradable polymers. He has authored more than 460 publications in SCI journals and issued over 110 patents. He has been awarded eleven National and Provincial Science \& Technology awards. In 2015, he was selected as an Academician of Chinese Academy of Engineering.

\section{物理、化学双重交联偶氮液晶聚合物的合成及其光热双重响应性能}

钟海艺 ${ }^{1,2}$, 陈力 $^{1^{*}}$, 丁晓敏 ${ }^{1}$, 杨荣 $^{3}$, 王玉忠 ${ }^{{ }^{*}}$

摘要 结合化学交联提供的稳定性和物理交联提供的可热塑加工性可设计得到具有多种刺激响应行为的新材料. 本文以偶氮苯二氧已醇 (BHHAB) 为液晶基元, 与苯基丁二酸 (PSA) 和丙三羧酸 (PTA) 本体聚合得到一系列液晶聚合物PBHPS- $x \%$ PTA, 其中PTA作为化学交 联, 而取代苯基与介晶基元之间的 $\pi-\pi$ 相互作用可提供物理交联. 热分析结果显示这些聚合物具有高的热稳定性并表现出近晶型液晶行 为. 广角X射线衍射 (WAXD) 和荧光光谱证实了分子间具有可作为物理交联点的 $\pi-\pi$ 相互作用; 并且随着PTA含量的增加, 化学交联会逐 渐影响分子链构象, 最终破坏物理交联作用. 轻度的化学交联保留了液晶聚合物的可热塑加工性, 而在物理交联与化学交联的共同作用下, 液晶聚合物具有可逆光致形变, 热致形状记忆及自修复性能, 在智能高分子材料领域表现出一定的应用前景. 